\title{
SCIDiC
}

International Journal of Forensic Science \& Pathology (IJFP)

ISSN: 2332-287X

\section{Dangerous Behaviors in a Patient with Mania: The Dilemma for Action where Mental Health Act is Lacking

Abdullah Aljaradi ${ }^{1}$, Mandhar Almaqbali ${ }^{*}$, Salim Al-Huseini ${ }^{3}$

${ }^{1}$ Sr. consultant psychiatrist, Armed Forces Hospital, Muscat, Oman.

${ }^{2}$ Specialist psychiatrists, Ministry of health, Sohar, Oman.

${ }^{3}$ Psychiatry Residency Program, Oman Medical Specialty Board, Muscat, Sultanate of Oman.

\section{Abstract}

Psychiatric disorders could impair the ability to make decisions and controlling impulses. This can result in dangerous behaviors that jeopardize the life of the patient and people around him. Lack of mental health act presents ethical and legal dilemma for further interventions in management of such patients. We report here a-47 years old patient with a severe manic episode associated with life-threatening behaviors who denied his symptoms and refused admission and treatment.

Keywords: Psychiatric Disorder; Dangerous Behavior; Manic Episode, Oman.

\section{Introduction}

Patients with severe psychiatric disorders bear a higher risk of violence than patients with other medical disorders [1]. Risk factors for violent behavior include young and male patients with diagnosis of bipolar disorder or schizophrenia [1]. Previous studies estimated the prevalence of violent behavior in patients with schizophrenia and bipolar disorder to be $8.2 \%$ and $12.4 \%$ respectively [1, 2]. In England and Wales, the annual incidence of homicidal convictions among patients with schizophrenia or other related delusion state between 2005 to 2015 was 6\% (NCISH 2017) [3]. Treatment of psychosis using antipsychotic medications and mood stabilizer is well documented in reducing violent and criminal behaviors in these patients [4]. Due to lack of insight and judgment impairment, patients with psychosis may refuse treatment and thus may bear risks on themselves and others. In Oman, legalization for compulsory treatment is not yet available.

\section{Case Presentation}

A 42-year old man brought to the psychiatry clinic by his brother for further evaluation and management of disturbed behavior for the past 2 months.

\section{History of Presenting Complaint}

He was noted to be over talkative and jumps from one topic to another with a loud speech. Also he overspends the family's financial resources on useless stuffs. In the clinic, he expressed ideas of inflated self-importance holding a belief that god had gifted him with extraordinary skills. He had no previous psychiatric background. His past medical history was significant for diabetes mellitus. He had no surgical history. The patient did not have a family history of psychiatric disorders. On mental status examination, the patient had full orientation. He was euphoric, talkative, appears overconfident and grandiose. His speech was pressured .He was agitated when he was interrupted. Sometimes he suddenly became angry. He had no hallucinations. He lacks the insight toward his illness. His workup included full blood count, complete metabolic panel, and thyroid function, all of which were unremarkable. Urine drugs screen was negative and his brain CT showed no abnormality. Using Diagnostic and Statistical Manual of Mental Disorders, 5th edition (DSM-5) criteria [5], he was diagnosed with bipolar disorder; current episode is mania with psychotic features. The patient was started on olanzapine $10 \mathrm{mg}$ by mouth at bedtimeand sodium valproate $400 \mathrm{mg}$ twice daily. He was advised for

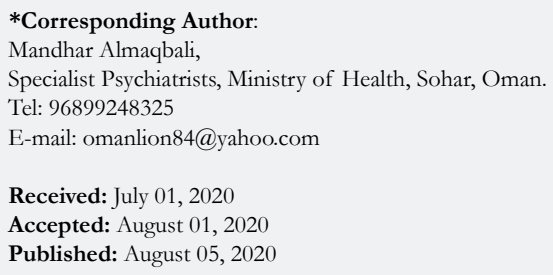

Copyright: Mandhar Almaqbali ${ }^{\circ}$ 2020. This is an open-access article distributed under the terms of the Creative Commons Attribution License, which permits unrestricted use, distribution and reproduction in any medium, provided the original author and source are credited. 
follow up after two weeks.

\section{Follow up/Reviews}

He did not show up for the follow up and his brother and wife came instead. He did not take his medications believing that he does not need them. He threatened to kill his wife with a knife. He was verbally abusive toward his children. He took his 10 -year old son in the car and drove with speed of $200 \mathrm{Km} / \mathrm{hr}$ and did not comply with the policemen who followed him. He went to his coworkers forcing them to adhere to his instruction as he is the most expert, culminated in a serious fight with them. He was contacted to attend the clinic for assessment. He presented in the second day with euphoric mood, flight of ideas, grandiose delusion and aggressive attitude. He refused all management options including admission and long acting antipsychotic injection. His family presented again after few days complaining that the patient is very violent at home. He threatened to kill them. He drives recklessly with high speeds exposing self and others to danger. The police was called for help but an official complaint and orders by public prosecution or court was required. The family was threatened to be killed if a complaint was made against him. Emergency psychiatry department of a tertiary care hospital for mental health was contacted to bring and admit the patient against his will. After legal department consultation, the mental health facility declined the request due to lack of legal cover for involuntarily assessment and admission. He injured his wife by a knife and kidnapped his younger son. The police was notified but the patient disappeared to unknown place.

\section{Discussion}

Involuntary admission is a common and controversial issue in psychiatric clinical practice and may raise clinical, ethical, and legal concerns [6]. Mental health act is a legalization that denotes assessment and treatment of patients with mental disorders under certain circumstances without their consent [7]. These circumstances are clearly defined in order for the patient to be "sectioned" under the mental health act. The ultimate goal of mental health act is to protect patients with severe mental disorders and people around them from imminent danger as well as assuring standards for mental health practice [7]. The number of patients admitted involuntarily in psychiatric hospital in England had increased by 20\% from 1996 to 2006 [8]. In Italy, 15.4\% of psychiatric inpatients were admitted involuntarily [9]. Psychotic disorders, suicidal acts and substance use disorders are the most common cause for compulsory admission under mental health act [10]. The patient outlined in this case report was clearly imposing imminent danger on himself by reckless driving for example and present an immediate threat to his family. Ambiguity upon the responsible facility privileged to compulsory assessand treat high-risk patients with psychiatric disorders resulted in delay in treatment. Injury of his wife and kidnapping his child could be prevented by early admission and treatment. In Oman, legalizations that guide mental health facility and law enforcement authority in such circumstances are not yet established. These legalizations will help in defining criteria for involuntary treatment and describe the role of mental health facility in situations where patients with psychiatric disorder bears threatened or real risk.

\section{Conclusion}

Patients with severe psychiatric disorders may present with disturbed behavior imposing risk on themselves and others. Lack of legalizations that guide mental health facility and law enforcement authority presents a significant challenge in management of such cases.

\section{References}

[1]. Swanson JW, Holzer III CE, Ganju VK, Jono RT. Violence and psychiatric disorder in the community: evidence from the Epidemiologic Catchment Area surveys. Hosp Community Psychiatry. 1990; 41(7): 761-70. PMID: 2142118.

[2]. Corrigan PW, Watson AC. Findings from the National Comorbidity Survey on the frequency of violent behavior in individuals with psychiatric disorders. Psychiatry research. 2005; 136(2-3):153-62.

[3]. Appleby L, Shaw J, Amos T. National confidential inquiry into suicide and homicide by people with mental illness. The British Journal of Psychiatry. 1997; 170(2):101-2.

[4]. Mohr P, Knytl P, Voráčková V, Bravermanová A, Melicher T. Long-acting injectable antipsychotics for prevention and management of violent behaviour in psychotic patients. International journal of clinical practice. 2017; 71(9). PMID: 28869705.

[5]. Edition F. Diagnostic and Statistical Manual of Mental Disorders. 5th. American Psychiatric Association. 2013.

[6]. Hoffmann K, Haussleiter IS, Illes F, Jendreyschak J, Diehl A, Emons B. Preventing involuntary admissions: special needs for distinct patient groups. Annals of General Psychiatry. 2017; 16(1):3.

[7]. Kelly BD. The Irish mental health act 2001. Psychiatric Bulletin. 2007 Jan; 31(1):21-4.

[8]. Keown P, Mercer G, Scott J. Retrospective analysis of hospital episode statistics, involuntary admissions under the Mental Health Act 1983, and number of psychiatric beds in England 1996-2006. Bmj. 2008; 337: a1837. PMID: 18845592.

[9]. Aguglia A, Moncalvo M, Solia F, Maina G. Involuntary admissions in Italy: the impact of seasonality. International journal of psychiatry in clinical practice. 2016; 20(4): 232-8.

[10]. Ellila HT, Sourander A, Välimäki M, Warne T, Kaivosoja M. The involuntary treatment of adolescent psychiatric inpatients-A nation-wide survey from Finland. Journal of adolescence. 2008; 31(3): 407-19. 